\title{
ON A FIXED POINT PROBLEM OF D. R. SMART
}

\author{
IRA ROSENHOLTZ
}

In his book, Fixed point theorems, D. R. Smart poses the following problem which he says appears to be open: "Does every shrinking (i.e. contractive) mapping of the closed unit ball in a Banach space have a fixed point?" We answer this question in the negative by exhibiting a contractive mapping from the closed unit ball in a Banach space to itself which has no fixed point. Furthermore, our mapping has the additional properties that it is affine, a homeomorphism onto its image, and its inverse is Lipschitz.

Recall that $C_{0}$ is the Banach space of all real sequences $x=\left(x_{1}, x_{2}, \ldots\right)$ such that $\lim _{n \rightarrow \infty} x_{n}=0$, and whose norm is defined by $\|x\|=\max \left\{\left|x_{n}\right|\right\}$.

We define our function as follows: Let $a_{1}, a_{2}, \ldots$ be any sequence of positive real numbers such that (i) each $a_{j}$ is less than 1 , and (ii) the sequence of partial products, $p_{n}=\prod_{j=1}^{n} a_{j}$, is bounded away from zero. (One such sequence is defined by $a_{n}=\left(2^{n}+1\right) /\left(2^{n}+2\right)$.) Now, if $x=\left(x_{1}, x_{2}, \ldots\right)$ $\in C_{0}$, we let $f(x)=\left(1, a_{1} x_{1}, a_{2} x_{2}, a_{3} x_{3}, \ldots\right)$. Then clearly if $\|x\| \leqq 1$, $\|f(x)\| \leqq 1$. (In fact, $\|f(x)\|=1$, if $\|x\| \leqq 1$.) Thus $f$ takes the unit ball in $C_{0}$ to itself. That $f$ is affine (i.e. that $f(t x+(1-t) y)=t f(x)+(1-t) f(y))$ is trivial. Next, notice that

$$
\|f(x)-f(y)\|=\max \left\{\left|a_{n}\left(x_{n}-y_{n}\right)\right|\right\}=a_{j}\left|x_{j}-y_{j}\right|,
$$

for some $j$, and if $x \neq y$,

$$
a_{j}\left|x_{j}-y_{j}\right|<\left|x_{j}-y_{j}\right| \leqq \max \left\{\left|x_{n}-y_{n}\right|\right\}=\|x-y\| .
$$

Therefore, since $\|f(x)-f(y)\|<\|x-y\|$ if $x \neq y, f$ is contractive.

Finally, suppose $x=\left(x_{1}, x_{2}, \ldots\right)$ is a fixed point of $f$. Then

$$
\begin{array}{ll}
x_{1}=1, & x_{3}=a_{2} x_{2}=a_{1} a_{2}, \\
x_{2}=a_{1} x_{1}=a_{1}, & x_{4}=a_{3} x_{3}=a_{1} a_{2} a_{3}, \text { etc. }
\end{array}
$$

and these numbers are bounded away from zero by the way that the sequence $a_{1}, a_{2}, \ldots$ was chosen. Thus, $x$ is not in $C_{0}$ and the proof is complete.

\section{REFERENCE}

1. D. R. Smart, Fixed point theorems, Cambridge Univ. Press, New York, 1974.

\section{Department of Mathematics, University of Wyoming, LaRamie, Wyoming 82071}

Received by the editors May 18, 1975.

AMS (MOS) subject classifications (1970). Primary 47H10, 54H25; Secondary 54E40, 46B99, $54 \mathrm{E} 50$. 